This is the accepted version of the following article: MacArthur C, Wilson D, Herbison P, Lancashire RJ, Hagen S, Toozs-Hobson P, Dean N, Glazener C, on behalf of the Prolong study group. Urinary incontinence persisting after childbirth: extent, delivery history, and effects in a 12-year longitudinal cohort study. BJOG 2015; which has been published in final form at DOI: $\underline{10.1111 / 1471-0528.13395}$

\title{
Urinary incontinence persisting after childbirth: extent, delivery history and effects in a 12 year longitudinal cohort study
}

C MacArthur ${ }^{a}$, D Wilson ${ }^{b}$, P Herbison ${ }^{c}$, RJ Lancashire ${ }^{a}$, S Hagen ${ }^{d}$, P Toozs-Hobson ${ }^{e}$, Nicola Dean ${ }^{\dagger}, \mathrm{C}$ Glazener ${ }^{\mathrm{g}}$ on behalf of the Prolong study group

${ }^{a}$ Public Health, Epidemiology and Biostatistics, School of Health and Population Sciences, University of Birmingham, Birmingham, UK

${ }^{b}$ Department of Women's and Children's Health and ${ }^{\mathrm{c}}$ Department of Preventive and Social Medicine, Dunedin School of Medicine, University of Otago, Dunedin, New Zealand

${ }^{d}$ NMAHP Research Unit, Glasgow Caledonian University, Glasgow, UK

${ }^{e}$ Birmingham Women's Hospital, Birmingham Women's NHS Foundation Trust, Edgbaston, Birmingham, UK

${ }^{\dagger}$ Department of Obstetrics and Gynaecology, York Hospital, Wiggington Road, York, UK

${ }^{9}$ Health Services Research Unit, University of Aberdeen, Aberdeen, UK

Correspondence: Prof C MacArthur, Public Health, Epidemiology and Biostatistics, School of Health and Population Sciences, University of Birmingham, Birmingham B15 2TT, UK. Email c.macarthur@bham.ac.uk, tel: 01214146770.

Short running title: Urinary incontinence persisting 12 years after birth 


\section{Abstract}

Objectives: To investigate extent of persistent urinary incontinence (UI) 12 years after birth and association with delivery mode history and other factors.

Design: Twelve year longitudinal cohort study.

Setting: Maternity units Aberdeen, Birmingham, Dunedin.

Population: Women who returned questionnaires 3 months and 12 years after index birth.

Methods: Data on all births over 12 months obtained from units then women contacted by post.

Main outcome measure: Persistent UI: reported at 12 years and one or more previous contact.

Results: Of 7879 women recruited at 3 months 3763 (48\%) responded at 12 years, 2944 also having responded at 6 years: non-responders had similar obstetric characteristics. Prevalence of persistent UI was 37.9\% (1429/3763). Among those who had reported UI at 3 months $76.4 \%$ reported it at 12 years. Women with persistent UI had lower SF12 scores. Compared to having only spontaneous vaginal deliveries (SVD), women who delivered exclusively by Caesarean section were less likely to have persistent UI (Odds Ratio (OR) $0.42,95 \% \mathrm{Cl} 0.33$ to 0.54 ). This was not the case in women who had a combination of CS and SVD births (OR 1.01, 95\% $\mathrm{Cl} 0.78$ to 1.30). Older age at first birth, greater parity, and overweight/obesity were associated with persistent UI. 46 (85.2\%) of 54 index primiparae with UI before pregnancy had persistent UI.

Conclusions: This study, demonstrating that UI persists to 12 years in about threequarters of women and that risk was only reduced with caesarean section if women had no other delivery mode, has practice implications.

Key words: Urinary incontinence; long term; postpartum; risk factors 


\section{Introduction}

Urinary incontinence $(\mathrm{UI})$ has been shown in many well designed studies to be common both in pregnancy and after childbirth with various risk factors identified, including delivery mode, parity, maternal age and Body Mass Index (BMI). ${ }^{1-3}$ Persistence of UI following childbirth in the longer term, however, is not well documented. A few large studies using birth registers have subsequently contacted women at a single point, up to 20 years after birth ${ }^{4-6}$ but evidence from cohorts of women who have been followed prospectively over time is sparse. Our longitudinal cohort study, which first contacted women 3 months after birth, found an overall prevalence of persistent UI at 6 years postpartum of $24 \%$. Most UI symptoms had not resolved and $73 \%$ of the women who had reported $\mathrm{UI}$ at 3 months also reported it at 6 years. $^{7}$ We have continued to follow our cohort to 12 years and have already reported that having $\mathrm{UI}$ at 12 years was less likely if a woman had all her births by caesarean section but not if the delivery history included other modes of birth. ${ }^{8}$ In addition to examining the prevalence of persistent $\mathrm{UI}$ and patterns of continuation and new onset, it is also important to investigate whether the same associations with delivery history and other obstetric and maternal factors are evident for persistent UI: and what the effects are on women's quality of life. These findings are presented in this paper.

\section{Methods}

All women who delivered during a 12 month period in 1993/1994 in three maternity units in Aberdeen (Scotland), Birmingham (England) and Dunedin (New Zealand) were sent a postal questionnaire at 3 months postpartum to assess prevalence of urinary and faecal incontinence. This is defined as the index delivery. Women with urinary incontinence at this time were eligible to take part in a randomised controlled trial $(\mathrm{RCT})$ of the effects of a home-based nurse-led pelvic floor muscle training 
(PFMT) programme (described elsewhere). ${ }^{9,10}$ All women who responded at 3 months were sent another questionnaire at 6 years postpartum and at 12 years, including non-responders at 6 years but excluding those who requested no further contact after 6 years and those known to have died.

\section{Data collection and outcomes}

To assess UI women were asked: “do you ever lose any urine when you don't mean to (yes/no)" and if yes, "in the last month how often has this happened on average" (response options ranged from less than twice per month to three or more times a day) and "do you wear a pad for this " (no, sometimes, all day, all night, all day and all night). A positive response to one or more of these questions was taken to indicate that the woman had UI. More severe UI was defined as occurring at least weekly. The questions were designed by the study team, since at the time of initial recruitment in 1993 there was no suitable validated questionnaire on UI and we continued with the same form of ascertainment in follow up for consistency. Questions were devised in accordance with the International Continence Society definitions. Women who reported UI were asked when it had first started in relation to their pregnancy and delivery (after this last delivery, during this last pregnancy, before this last pregnancy). Types of UI were ascertained by asking about loss of urine 'when you cough, laugh, sneeze, run, jump or play sport (stress UI); feel an urgent desire to pass water/urine and unable to reach the toilet on time (urgency UI) or at some other time (other). Types of UI were categorised into stress without urgency, urgency alone, mixed stress and urgency and other UI. Further questions were about the extent to which UI interfered with everyday life (on 10 point scale from 'not at all' to 'a great deal') and whether in the past, they had had any drug, physiotherapeutic and/or surgical treatments (other than that associated with our earlier study in $1993 / 4)^{9,10}$ for their UI. 
The SF12 was included in the 12 year questionnaire to assess general health-related quality of life. This 12 item tool has two summary scores, a mental component score (MCS) and a physical component score (PCS), to assess mental and physical functioning, both with a range of $0-100$ and a mean of 50 , higher scores indicating better function. ${ }^{11}$

The main outcome of persistent UI was defined as UI reported at 12 years as well as at least one of the previous contact points of 3 months or 6 years.

Obstetric and maternal data on the index delivery were obtained from the hospital case-notes. Follow-up questionnaires at 6 and at 12 years obtained date and mode of every delivery from each woman to determine delivery mode history. The initial study and both follow-ups were approved by ethics committees in each centre.

\section{Research questions and analysis}

The main research questions investigated in this paper were the extent and pattern of postpartum UI persistence and whether delivery mode history and other factors are predictive. The relationship between persistent symptoms and quality of life was a secondary research question and prevalence of new onset $\mathrm{UI}$ across contacts was also of interest.

The variable delivery mode history was created from the reported birth histories at 12 years. This categorised all of a woman's deliveries, including those which pre-dated our first contact with them 3 months following the index birth as: spontaneous vaginal delivery (SVD) only; Caesarean Section (CS) only; one or more forceps; one or more vacuum extraction but no forceps; a combination of SVD and CS. 
Multiple logistic regression was used to investigate independent associations between delivery mode history and outcomes, adjusting for age at first birth $(<25 / 25-$ 29/30-34/35+), total number of births (one/two/three/four or more), current BMI (underweight, normal weight, overweight, obese and not known) and ethnic origin (non-Asian/Asian): and to report on other independent associations.

More detailed obstetric factors from case-notes were only available for index births, so to investigate further obstetric associations, a sub-group analysis was undertaken, restricted to women for whom the index birth was their first (referred to as index primiparae). Additional variables included were onset of labour (not induced/induced), perineal trauma (intact/episiotomy/laceration), and birthweight (quartiles). Degree of laceration was not recorded in a comparable format from all three units to allow separate investigation of $3^{\text {rd }}$ or $4^{\text {th }}$ degree tears. Cases with missing values (except BMI - see above) were omitted from the models.

\section{Results}

$3763(48 \%)$ of the women recruited to the cohort at 3 months postpartum responded at 12 years. Among these 3763 women, 2944 (78.2\%) had replied at all three contact points. Respondents and non-respondents at 12 years were similar in their main obstetric characteristics and 3 month UI prevalence but there were some demographic differences. Details of response rate and responder/non-responder comparisons are given elsewhere. ${ }^{8}$

\section{Prevalence and pattern of persistent UI}

The prevalence of persistent $\mathrm{UI}$ at 12 years (i.e. $\mathrm{UI}$ at 12 years plus one other contact) was $37.9 \%(1429 / 3763)$. Table 1 shows details of the pattern of UI across the three contact points of 3 months, 6 years and 12 years postpartum for all women; and separately for women who had given birth to their first baby at our first contact 
with the cohort at 3 months (1760 index primiparae). Table 1 also separately distinguishes responses from women who replied at all of the three contacts or at just 3 months and 12 years. Among the group who had replied every time, $21.2 \%$ $(625 / 2944)$ had reported UI at every contact. Among all women who responded at 12 years, a total of $33.1 \%(1247 / 3763)$ had reported UI at 3 months, whilst at 6 years it had been $46.8 \%(1378 / 2944)$ and $52.7 \%(1983 / 3763)$ at 12 years. For the index primiparae who responded at 12 years, the proportions with persistent $\mathrm{UI}$ and with $\mathrm{UI}$ at the various contact points were similar $(29.5 \%, 45.0 \%$ and $50.7 \%$ respectively) to those among women of all parities (Table 1).

In women who had reported UI at 3 months, symptom resolution over the 12 years was not common, with UI persisting in most cases: in the whole sample, $76.4 \%$ of the women with UI at 3 months also reported UI at 12 years (953/1247)

(Table 1). Among the group of 2944 women who had replied at all three contact times, $64.6 \%$ of those with UI at 3 months (625/967) reported it again at both 6 and 12 years: only $13.5 \%$ (131/967) did not subsequently report UI at all. Looking separately at the 1373 women who had given birth for the first time at our 3 month contact (index primiparae) and replied every time, the pattern of UI persistence and resolution was similar to that among women of all parities (Table 1).

In addition to persistence, there was new onset of UI in women who had been 'dry' at our 3 month contact. Among the 2944 women who responded all three times, 32.8\% had UI at 3 months (967/2944), then $33.1 \%$ of the remaining 'dry' women $(655 / 1977)$ had UI at 6 years and $25.3 \%$ (335/1322) of those 'dry' at 6 years had UI at 12 years. For the index primiparae who replied all three times the pattern was similar: the proportion with $\mathrm{UI}$ in these primiparae had been $29.9 \%(410 / 1373)$ at 3 months and $32.9 \%$ had new onset UI at 6 years among those previously 'dry' (317/963), and $25.9 \%$ of those dry at 6 years $(167 / 646)$ had UI at 12 years. Over half $(55.1 \%$, 
$389 / 705$ ) of the consistently responding index primiparae who had UI at 12 years had not had UI following their first birth. $69.7 \%(957 / 1373)$ of the consistently responding index primiparae had had another baby between our 3 month and 12 year contacts.

Our question at the 3 month contact on whether their UI had started pre index pregnancy did not allow us to distinguish between UI before the index or some previous pregnancy in those who were multiparae at first contact. However, among the 1760 for whom the index pregnancy was their first, only 54 (3.1\%) had reported a pre-pregnancy onset of UI of whom $46(85.2 \%)$ had persistent UI at 12 years.

Severity and effects

Most of the women who reported persistent UI reported it as occurring less often than daily at 12 years, with only $14.3 \%$ (204/1429) reporting it occurring daily or more. There was, however, indication of some severe symptoms: $40.9 \%(584 / 1429)$ of the women with persistent $\mathrm{UI}$ said at 12 years that they used a pad to protect against leakage and $14.5 \%(207 / 1429)$ said they wore a pad all the time. In terms of interference with everyday life only $5.5 \%$ of the women with persistent UI (79/1429) reported no effect at all (on a scale $0-10$ where 10 is a great deal) and $37.2 \%$ $(532 / 1429)$ rated it as 5 or more. Half of the women with persistent UI reported an effect on hygiene (713/1429); 19\% (266/1429) on home life; $27 \%(322 / 1214$ who worked) on work life, $45 \%$ on social life, and $18 \%$ (226/1241 with a partner) on sex life. Even though there were 204 women in the sample with persistent and daily UI, only 18 women said that they had had any treatment for their UI: six women said that they had only drug treatment, six had drug and physiotherapy and six had surgical treatment in addition to drugs and physiotherapy.

The quality of life SF12 Mental Component Score (MCS) and Physical Component Score (PCS) were significantly lower (worse) among women with persistent UI than 
without (Table 2). Given that women without persistent UI comprised three subgroups (UI at 12 years but not before; UI sometime since index birth but not at 12 years; no UI at all) we looked separately at mean MCS and PCS scores in these subgroups. This showed that those who had not had UI and those who had previous UI that had now resolved had significantly higher (better) SF12 scores than those who had persistent $\mathrm{UI}$ at 12 years (Table 2). There were no significant differences between the sub-group that reported $\mathrm{UI}$ at 12 years and not before relative to those who had persistent UI.

\section{Persistent urinary incontinence and delivery mode history}

Persistent UI was significantly less common if all of a woman's births were by caesarean section relative to all SVD births but there was no association between persistent UI where the delivery history comprised a mix of SVD and CS births (Table 3). There was an independent association between persistent UI and older maternal age at first birth and increasing parity. Current BMI also showed an independent association: both overweight and obese women had significantly more persistent UI compared with those of normal weight (Table 3). We repeated the model, separately categorising type of caesarean section into pre-labour, post labour or both: the reduced likelihood of persistent UI for exclusive caesarean birth was similar for all types of sections (see Table 3 footnote) and the other independent associations remained the same. The comparison shown in Table 3 is between women with and those without persistent UI and the latter included women who had UI that was not persistent (i.e. at 3 months and/or 6 years but not 12 years) as well as those who had not had UI at all. We therefore repeated the logistic regression model excluding from the analysis all women who had experienced non-persistent UI and the same pattern of associations remained (data not shown). 
The model was repeated for primiparae at index birth to consider other obstetric factors, none of which were significantly associated with persistent UI (Table 4). The reduced likelihood of persistent UI with only CS births remained with entry of the additional obstetric factors into the model.

\section{Types of UI}

Among the women with persistent UI (54.2\%: 775/1429) had stress without urgency incontinence, 32.8\% (469/1429) had mixed stress and urgency, 9.8\% (140/1429) had urgency alone, 2.5\% (28/1429) had some other incontinence and 17 did not provide information to categorise type. Table 5 shows this according to delivery mode history. Patterns appear similar for stress UI with and without urgency. Although the numbers for urgency alone were small, a similar UI reduction in women who only delivered by CS relative to those who only delivered by SVD however does not seem to be present.

\section{Discussion}

Main findings

This large cohort study, following women 3 months, 6 and 12 years after birth, has shown $37.9 \%$ prevalence of persistent UI. At least two-thirds and up to threequarters of women with UI that was present at 3 months still had it at 12 years. Caesarean delivery only protected from persistent UI if a woman had no other delivery mode and to our knowledge this is the first study to report this.

Although $85 \%$ of women with persistent UI reported less than daily occurrence, this did impact on their lives, with over $40 \%$ of these women reporting some pad use, almost half an effect on their social life and over a quarter on work life. SF12 MCS and PCS quality of life scores were significantly worse in women with persistent UI, although the clinical significance of these reduced scores is not known. Even with 
over 200 women reporting at least daily UI, a very low proportion of symptomatic women presented for drug, physiotherapeutic or surgical treatment.

This is one of the first studies to document extent of new onset UI. Among index primiparae with UI at 12 years who replied every time, two-thirds did not have UI following their first birth, but over $40 \%$ of these initially 'dry' women had it at 12 years.

Strengths and limitations

The large size, duration and successive follow-up are the main study strengths. It is one of the largest postpartum cohort studies with successive long term follow-up. Its size allowed sufficient women in the various delivery mode history groups for comparisons of persistent UI to be made with acceptable precision.

Obstetric data for index delivery were available in case notes, although delivery history was obtained directly from women, a possible limitation. Previous studies, however, have shown that women's reports of delivery details are highly accurate. ${ }^{12}$ We checked women's reports of index delivery mode with case notes and showed that $98 \%$ of CS, $98 \%$ of SVD, $87 \%$ of forceps and $88 \%$ of vacuum deliveries were consistent. Another weakness is that a validated UI questionnaire was not used but at the time of recruitment in 1993 none was available and we wanted to follow-up using consistent questioning. BMI was based on self-reported height and weight, potentially subject to bias, but there were no other options with such a large cohort.

Follow-up rate at 12 years may be considered the main study limitation, at $50 \%$ of those initially contacted at 3 months. However, it is important to consider ways in which this response rate might produce bias. The initial study intention had been for a single 3 month contact so we did not seek alternative contacts in event of house move. At 6 years alternative contact details were sought and attrition between 6 and 
12 years was much smaller, with 70\% of 6 year responders responding again. By 12 years, additional tracing methods were also available in UK centres and 819 women who had moved by 6 years, thus not responded, did so when traced to a different address. It is, therefore, likely that much non-response was from geographical mobility, not known to be systematically associated with incontinence or delivery mode. We had index birth case-note data for all women and comparisons were made between responders and non-responders at 12 years to examine response bias. Fewer women under 25 and from ethnic minority groups responded, a typical response pattern among childbirth populations. ${ }^{13}$ Younger women had less UI, thus the age difference is likely to lead to overestimate of UI prevalence. Obstetric factors, however, particularly delivery mode, were broadly similar between responders and non-responders: and it difficult to see how respondent demographic differences might bias UI delivery mode associations.

Interpretation

Exclusive caesarean delivery history relative to only SVD births was independently associated with reduced likelihood of persistent UI. However, women who delivered by a combination of CS and SVD incurred the same risk as only SVD births. Type of CS in the delivery history, exclusively pre-labour, exclusively post-labour or a mix of both showed similarly reduced risks of persistent UI. Previous studies of UI following only one birth have shown no distinction in 'protection' from different types of CS, consistent with our findings. ${ }^{5,14}$ We were only able to sub-divide CS into all prelabour (elective), all post-labour (emergency) or both but a US study ${ }^{14}$ of first births was able to further sub-divide post-labour sections into those with and without pushing, and found similar protection from UI for both 3-6 months later.

Our main analysis included all types of UI but additional analysis separating type showed protection with exclusive CS births only for stress UI (+/- urgency) but not 
urgency $\mathrm{UI}$ alone, although the latter group was small. This is consistent with the findings of the Norwegian EPINCONT study of UI at a single point 30 years after birth in women who had delivered only by vaginal delivery or only by caesarean section. ${ }^{5}$ It is inconsistent however with a Swedish study that contacted women recorded in a birth registry as only ever having one birth, and showed all types of UI as less prevalent after one caesarean than one vaginal delivery. 5,15

Overweight and obesity at 12 years was associated with persistent UI, as was older age at first birth and greater parity. These associations with persistent $\mathrm{UI}$ are consistent with the literature on $\mathrm{UI}$ at a single time point, in the short ${ }^{14}$ and longer term since birth. ${ }^{3,4}$ The Swedish study, found that higher BMI was strongly associated with more severe UI symptoms. ${ }^{15}$ There is little literature documenting risk factors of persistent $\mathrm{UI}$ from populations with successive follow-up. Our study found few ( 3\%) women reporting UI starting before first pregnancy, but consistent with shorter follow-up studies, ${ }^{16}$ this strongly related to persistent UI.

There is little information on impact of postpartum UI on quality of life and we found none relating to persistent $\mathrm{UI}$ several years after birth. A US study of UI in primiparous women and 6 month quality of life found worse SF12 PCS and MCS scores in women with UI than those without. ${ }^{17}$ They found no relationship between SF12 scores and severity of UI nor differences according to UI type.

\section{Conclusions}

This large longitudinal study demonstrating that two-thirds to three-quarters of women with $\mathrm{UI}$ after birth have persistent urinary incontinence many years later is important for advice and practice. In discussions about caesarean section choice, medical advisors inform to women that although exclusive CS birth is protective of persistent UI it only offers partial protection and repeated caesareans are associated 
with other adverse effects. And women that have a caesarean section as well as other delivery modes have no protection from UI. Excessive weight is potentially modifiable and the overweight/obesity relationship with persistent UI is important. Implementation of antenatal prevention strategies are urgent, in particular correctly practised pelvic floor muscle exercises where there is evidence of at least short term benefit. That UI commonly persists might motivate both clinical advisors and women in their importance. Although persistent UI was associated with lower quality of life almost no women reported treatment resulting in major service unmet need.

\section{Word Count: 3548}

\section{Disclosure of interest}

All authors declare that they have no interests to declare.

\section{Contribution to authorship}

$\mathrm{CM}, \mathrm{CG}, \mathrm{DW}, \mathrm{RL}$ and PH contributed to design and analysis of the whole ProLong study. SH, ND and PT-H joined the study group at 12 years and contributed to this part of the study. CM drafted the paper with CG and all authors commented. Other members of the ProLong study group are Adrian Grant and Christine Bain.

\section{Details of ethics approval}

Ethical approval for UK centres for 12 year follow up was obtained from Multicentre Research Ethics Committee Edinburgh, Ref No RG 819/06, November 2007 and from New Zealand National Ethics Committee, Ref No LRS/05/04/009 March 2005.

\section{Funding}

Grants were obtained from Wellbeing of Women/Royal College of Obstetricians and Gynaecologists for the UK follow up and from the Health Research Council of New Zealand for follow up in New Zealand.

\section{Acknowledgements}

The authors thank the women who took part in the study and Alison McDonald, Anne-Marie Rennie, Jane Cook and Jane Harvey who were involved in assembling 
the original cohort. Gladys McPherson was responsible for the database design and Margaret MacNeil was involved in the administration of the follow up study.

\section{References}

1. Brown SJ, Donath S, MacArthur C, McDonald EA, Krastev AH. (2010). Urinary incontinence in nulliparous women before and during pregnancy: prevalence, incidence, and associated risk factors. Int Urogynecol J 21:193-202.

2. Nygaard I, Barber M, Burgio K, et al Prevalence of systematic pelvic floor disorders in US women. JAMA 2008;300:1311-6.

3. Rotveit G, Hunskaar S. Urinary incontinence and age at the first and last delivery: The Norwegian HUNT/EPINCONT study. Am J Obstet Gynecol 2006;195:433-8.

4. Dolan LM, Hilton P. Obstetric risk factors and pelvic floor dysfunction 20 years after first delivery. Int Urogynecol J Pelvic Floor Dysfunct 2010;2:535-44

5. Gyhagen M, Bullarbo M, Nielsen TF, Milsom I. The prevalence of urinary incontinence 20 years after childbirth: a national cohort study in singleton primiparae after vaginal or caesarean delivery. BJOG 2012;120:144-51.

6. Rortveit G, Dalveit AK, Hannestad YS, Hunskaar S; for the Norwegian EPINCONT Study. Urinary Incontinence after vaginal delivery or cesarean section. N Engl J Med 2003;348:900-7.

7. MacArthur C, Glazener CMA, Wilson PD, Lancashire RJ, Herbison GP, Grant AM. Persistent urinary incontinence and delivery mode history: a six-year longitudinal study. BJOG 2006;113:218-24.

8. MacArthur C, Glazener C, Lancashire RJ, Herbison P, Wilson D; ProLong Study Group. Exclusive caesarean section delivery and subsequent urinary and faecal incontinence: a 12-year longitudinal study. BJOG 2011;118(8):1000-7. 
9. Glazener CMA, Herbison GP, Wilson PD, MacArthur C, Lange GD, Gee H, et al. Conservative management of persistent postnatal urinary and faecal incontinence: randomised controlled trial. BMJ 2001;323:593-6.

10. Glazener CMA, Herbison GP, MacArthur C, Grant A, Wilson PD. Randomised controlled trial of conservative management of postnatal urinary and faecal incontinence: six year follow up. BMJ 2005;330:337-9.

11. Ware J, Kosinski M, Turner-Bowker D, Gandek B. How to score version 2 of the SF12 health survey. Lincoln RI; QualityMetric Incorporated. 2002.

12. Boyles SH, Li H, Mori T, Osterweil P, Guise JM. Effect of mode of delivery on the incidence of urinary incontinence in primiparous women. Obstet Gynecol 2009;113(1):134-41.

13. Gyhagen M, Bullarbo M, Nielsen TF, Milsom I. A comparison of the long-term consequences of vaginal delivery versus caesarean section on the prevalence, severity and bothersomeness of urinary incontinence subtypes: a national cohort study in primiparous women. BJOG 2013;120:1548-1555.

14. Brown S, Donath S, MacArthur C, McDonald E, Krastov A, Urinary incontinence in nulliparous women before and during pregnancy : prevalence, incidence, and associated risk factors. Int Urogynecol J 2010;21(2);193-202.

15. Handa VL, Zyczynski HM, Burgio KL, Fitzgerald MP, Borello-France D, Janz NK, et al; for the Pelvic Floor Disorder Network. The impact of fecal and urinary incontinence on quality of life 6 months after childbirth. Am J Obstet Gynecol 2007;197(6):636.e1-6.

16. Traude P, L'He' lias LF, Raison-Boulley A-M, Castel C, Pichon C, Bouyer J, et al. Perinatal factors reported by mothers: do they agree with medical records? Eur J Epidemiol 2008;23:557-64.

17. Cartwright A. Who responds to postnatal questionnaire? J Epidemiol Community Health 1986;3:267-73. 